\title{
A snapshot of environmental health conditions among indigenous Baram communities at Borneo, Sarawak
}

\begin{abstract}
Background: This paper outlines the environmental health conditions arising from the synergistic effect of the rampant human activities (illegal logging and rapid expansion of oil palm plantations) that are exploiting the nature resources without proper planning. This led to the unsolved health problem caused by multifaceted of hazards among the remote indigenous Baram communities at Borneo, Sarawak. Methodology: This is an observational survey, which conducted in two phases, that is, June 2014 and January 2016. A random sampling of locations from the downstream and reservoir zones of the Baram River were selected for site surveillance. A total of 10 indigenous villages were visited and 3 main populations of affected indigenous people (Kayan, Kenyah, and Penan) were recruited for short interviews. Result: The result showed that at least $80 \%$ of the interviewed villagers held clear views on the identified environmental hazards; however, site surveillance to review the environmental health conditions of their living area showed that the health of the indigenous community is in the vulnerable and precarious conditions. Discussion and Conclusion: This observational survey provides insights of the current environmental health conditions among the remote aboriginal communities at Borneo Island. The data provides a detailed picture of the state of environmental health conditions and discuss the potential health consequences among the indigenous communities across Baram communities.
\end{abstract}

Keyword: Environmental health; Health condition; Indigenous; Inequality; Remote 